\title{
Photoemission current-spacecraft voltage relation: Key to routine, quantitative low-energy plasma measurements
}

\author{
J. D. Scudder and Xuejun Cao \\ Department of Physics and Astronomy, University of Iowa, Iowa City \\ F. S. Mozer \\ Space Sciences Laboratory, University of California, Berkeley
}

\begin{abstract}
Measurements of the floating potential and plasma return current on the GGSPolar spacecraft are used to determine the equilibrium photoemission current, $J_{\hbar v}$, as a function of the spacecraft's $(\mathrm{S} / \mathrm{C})$ "floating potential," $\Delta \Phi_{\mathrm{S} / \mathrm{C}}$. The photoemission current function is found to be time independent using nearly 10 months of GGS-Polar data from April 1996 to March 1997 including 1.6 million separate spectra from the Hydra (hot plasma) and EFI (electric field) instruments. The photoemission current density leaving the spacecraft at positive floating potentials $\Delta \Phi_{\mathrm{S} / \mathrm{C}}<50 \mathrm{~V}$ is well fit by a sum of two exponentials of the form: $J_{\hbar \nu}\left(\mu \mathrm{A} / \mathrm{m}^{2}\right)=A \exp \left(-\Delta \Phi_{\mathrm{S} / \mathrm{C}} / B\right)+C \exp \left(-\Delta \Phi_{\mathrm{S} / \mathrm{C}} / D\right)$, where best fit estimates $A \simeq 152 \pm 57 \mu \mathrm{A} / \mathrm{m}^{2}, B \simeq 1.7 \pm 0.2 \mathrm{eV}, C \simeq 0.86 \pm 0.29$ $\mu \mathrm{A} / \mathrm{m}^{2}$, and $D \simeq 9.5 \pm 1.0 \mathrm{eV}$. In equilibrium this photoemission current density is determined from the ratio of sunlit to spacecraft areas and the plasma current density, $J_{R C}$, collected by the spacecraft. For the Polar spacecraft this ratio of areas is not constant in time, and the observed return current voltage relation is time dependent. After correction for the orbitally induced time-dependent ratio of areas, the photoemission curve reported above is obtained and is essentially time independent. After correcting for the different procedures used the present results are illustrated to be consistent with early results with less resolution reported by Pedersen [1995]. When the sensing of the floating potential by EFI on the spacecraft is interrupted, the photoemission-voltage relationship is essential for the assignment of the ambient kinetic energy of the detected particle fluxes. We demonstrate a method using the plasma data and the statistical return current relationship that recovers the floating potential of the spacecraft with a typical precision that is the larger of $0.5 \mathrm{~V}$ and $0.1 \Delta \Phi_{\mathrm{S} / \mathrm{C}}$. We also demonstrate that the ion and electron densities determined by numerical integration over distributions corrected with opposite energy shifts implied by the potential enhances their routine agreement, a further check on the absolute precision of the potential inferences. The "systematic" departures of $40 \%$ of the data from the statistically defined $J_{\hbar \nu}\left(\Delta \Phi_{\mathrm{S} / \mathrm{C}}\right)$ curve reported above are time varying and organized in radius and with $L$ shell. These data are inferred to be the signature of missing ambient plasma currents to the spacecraft that are not directly detected by the Hydra instrumentation (cf. X. Cao et al., Properties of very cold $\left(T_{e} \simeq 0.1 \mathrm{eV}\right.$ ) electrons within the magnetosphere, submitted to Journal of Geophysical Research, 1999).
\end{abstract}

\section{Introduction}

As the sophistication of space plasma measurements increase, and the detection energy of electron and ion measurements approach those of the typical floating potential of the vehicles in space, the routine in flight determination of the potential and its variations are essential for high-precision derived plasma properties. This should be clear because the phase space density observed at collected kinetic energy $E^{\prime}$ reflects information about the phase space density that had energies $E^{\prime}+q \Delta \Phi_{\mathrm{S} / \mathrm{C}}$ well outside the Debye layer sheath about the vehicle, where $\Delta \Phi_{\mathrm{S} / \mathrm{C}}$ is the electrical potential difference of the spacecraft surface with respect to the nearby plasma. These concerns are particularly relevant for the determination of accurate electron and low energy ion densities,

Copyright 2000 by the American Geophysical Union.

Paper number 1999JA900423.

0148-0227/00/1999JA900423\$09.00 number fluxes, and current densities with particle detectors in plasmas with low mean energies. Accurate electron flow velocities are central both to the in situ current density determinations and the inference of the motion of magnetic field lines. To determine an accurate electron flow velocity on a spacecraft is challenging enough without imprecise information about the floating potential.

In addition to establishing the ambient energy of collected particles outside the spacecraft sheath, the routine knowledge of the spacecraft's floating potential aids the removal from the observed electron spectrum of photoelectrons trapped within the spacecraft sheath that meet the inequality $E^{\prime}<|e| \Delta \Phi_{\mathrm{S} / \mathrm{C}}$. Within the magnetosphere there are a variety of low-energy ( $T \simeq 1 \mathrm{eV}$ ) electron populations that are not photoelectrons caused by the interaction of the solar UV spectrum with the spacecraft surface. The best chance of knowing that observed low temperature electron populations are geophysical comes from using the measured $\Delta \Phi_{\mathrm{S} / \mathrm{C}}$ together with the knowledge 
of its precision to remove trapped spacecraft photoelectrons from the observed spectrum. The recovery of a timeindependent photoemission curve with our data analysis has led to a variety of understandings and clarifications of both the quality of the floating potential information and its precision which set the limits for the future discovery process of cold plasmas in the magnetosphere and the precision of odd moments from the electrons.

In the past, plasma experimentalists have incorporated a variety of special hardware modes or ground analysis in attempts to correct for the effects of spacecraft potential variations. Rosenbauer et al. [1976], Bame et al. [1978], and Paterson et al. [1998] describe special hardware modes to sense the presence of photoelectrons by the break in the spectrum.

Knowledge a priori of the low-energy form of the ambient plasma distribution was used by Feldman et al. [1975] to infer the size of the potential as a fitting parameter. Scudder et al. [1981] used quasi-neutrality between supersonic ion measurements with little sensitivity to floating potential and electron data in the solar wind on the same spacecraft to develop an empirical photoemission spectrum for subsequent use within Jupiter's magnetosphere. A similar approach had been used to determine the floating potential and the plasma return current for use in iterating the floating potential variation on the ISEE 1 vehicle (J. D. Scudder and F. S. Mozer, private communications, 1977-1978).

In this paper we describe the implementation of a return current technique similar to those used previously on ISEE 1 and Voyager, tailored for the GGS-Polar spacecraft that uses the routine determinations of the floating potential from the EFI experiment [Harvey et al., 1995] and the plasma return current derived from the data of the Hydra plasma experiment [Scudder et al., 1995]. We show the results of this construction and illustrate how it may be used to infer the potential when the onboard estimates of $\Delta \Phi_{\mathrm{S} / \mathrm{C}}$ from EFI are not available. This situation usually occurs in warm plasmas of high densities which are found in the cusp and at the magnetic equator. The cusp proper is a location where the determination of accurate, vector electron and ion plasma moments, including current signatures and occasional direct signatures of ongoing reconnection, are an ongoing scientific objective of the Polar mission. The advent of high-quality electron flow parameters that can be determined when $\Delta \Phi_{\mathrm{S} / \mathrm{C}}$ is accurately known, can be used to statistically validate the cross-field drift velocity of the plasma in terms of the $c(\mathbf{E} \times \mathbf{B}) / B^{2}$ velocity determined by three-axis $\mathbf{B}$ and $\mathbf{E}$ measurements.

\section{Physics of the Spacecraft's Floating Potential}

Consider an initially uncharged, conducting surface (that will become the spacecraft subscript $\mathrm{S} / \mathrm{C}$ in the discussion that follows) that is immersed in a plasma. Because the plasma electron thermal velocity is typically greater than that of the ions, more plasma electrons than ions strike this surface, so it initially collects a negative current from the plasma. If the surface is in sunlight, it also emits photoelectrons. If the plasma is "underdense," the electron current from the plasma is less than the photoelectron current, so the conducting surface starts charging positively. As it charges more positive, it attracts back some of the otherwise escaping photoelectrons that were released from its surface, so that the net charging rate decreases. Eventually, an equilibrium is reached in which the magnitude of the incoming plasma current just equals that of the escaping photoelectrons able to overcome the potential difference between the charged surface and the nearby plasma, denoted by subscript $p$, and to thereby escape to infinity. The equilibrium potential difference $\Delta \Phi_{\mathrm{S} / \mathrm{C}}$ between the conducting surface and the nearby plasma is determined by this current balance condition and is almost always positive in sunlight.

To simplify the following derivation while retaining the leading terms, assume that the ion current from the plasma is negligible compared to the electron return current $\mathrm{RC}$ denoted by $I_{\mathrm{RC}}$, from the plasma. Also, assume that the photoelectron energy spectrum is a pseudoexponential with an $e$ folding energy, $W_{0}$, that can depend on the energy of observation. If, in addition, it is assumed that every plasma electron that strikes the conductor sticks to it and that the secondary emission is small, the current balance condition becomes

$$
I_{R C}-I_{\hbar \nu} \exp \left[-\frac{|e|\left(\Phi_{\mathrm{S} / \mathrm{C}}-\Phi_{p, \mathrm{~S} / \mathrm{C}}\right)}{W_{0}\left(\Phi_{\mathrm{S} / \mathrm{C}}-\Phi_{p, \mathrm{~S} / \mathrm{C}}\right)}\right]=0,
$$

where $I_{\hbar \nu}$ is the total photoelectric current from the conductor. The potential difference between the conducting surface and the nearby plasma, $\Delta \Phi_{\mathrm{S} / \mathrm{C}} \equiv \Phi_{\mathrm{S} / \mathrm{C}}-\Phi_{p, \mathrm{~S} / \mathrm{C}}$, is sometimes (loosely) referred to as the "floating" potential of the conducting surface.

Although (1) provides a relationship between the plasma parameters that define $I_{R C}$ and the potential difference between the conductor and the nearby plasma, it cannot be applied directly to experimental data because the potential difference $\Delta \Phi_{\mathrm{S} / \mathrm{C}}$ between the conducting surface and the nearby plasma cannot be measured directly. To overcome this problem, consider a second conducting surface denoted by subscript $s$ (which is a sphere on a boom away from the spacecraft body associated with the electric field experiment, EFI) that has an additional bias current provided by electronics that is connected between surfaces $s$ and main body of the spacecraft $\mathrm{S} / \mathrm{C}$. Current balance for surface $s$ thus becomes

$$
I_{R C}-I_{\hbar v} \exp \left[-\frac{|e|\left(\Phi_{s}-\Phi_{p, s}\right)}{W_{0}\left(\Phi_{s}-\Phi_{p, s}\right)}\right]+I_{\text {bias }}=0 .
$$

If the current $I_{\text {bias }}$ is negative, the potential difference between surface $s$ and its nearby plasma is reduced in magnitude. When $I_{\text {bias }}=-\left(I_{R C}-I_{\hbar \nu}\right)$, the potential difference between surface, $s$, and the nearby plasma (in the numerator of (2)) is 0 . To be safe in practice, the bias current is set to somewhat less than this value so the surface, $s$, is one or two volts positive, $\Delta \Phi_{\text {bias }}$ with respect to its nearby plasma, i.e.,

$$
\Phi_{s}=\Phi_{p, s}+\Delta \Phi_{\text {bias }}
$$

where $\Delta \Phi_{\text {bias }}$ is 1 or $2 \mathrm{~V}$.

Because the sphere potential is near plasma potential, most of its emitted photoelectrons escape. This current must be collected by the spacecraft in order to maintain overall current equilibrium. It appears that an extra small term has been neglected in (1). In fact, this is believed not to be the case because the escaping photoelectrons from the spheres are attracted by the positive potentials on the outer braids of the sphere cables (which are at spacecraft potential) so they are collected without the need for the spacecraft potential to change.

Under the assumption that the potential difference between the plasma near the sphere and the plasma near the spacecraft, $\left(\Phi_{p, s}-\Phi_{p, \mathrm{~S} / \mathrm{C}}\right)$, is small then, using equation (3), 


$$
\begin{gathered}
\Phi_{p, s} \simeq \Phi_{p, \mathrm{~S} / \mathrm{C}} \\
\Delta \Phi_{\mathrm{S} / \mathrm{C}}=\Phi_{\mathrm{S} / \mathrm{C}}-\Phi_{p, \mathrm{~S} / \mathrm{C}} \simeq \Phi_{\mathrm{S} / \mathrm{C}}-\Phi_{p, s} . \\
=\Phi_{\mathrm{S} / \mathrm{C}}-\Phi_{s}+\Delta \Phi_{\text {bias }}
\end{gathered}
$$

(The potential associated with the electric field between two points in the sheath near the spacecraft is very small relative to the engineering voltages that are being sensed.) Because the spacecraft body's potential relative to that of the spherical probe, $\left(\Phi_{\mathrm{S} / \mathrm{C}}-\Phi_{s}\right)$, can be measured directly, and because $\Delta \Phi_{\text {bias }}$ can be estimated from knowledge of the EFI sphere's photoemission and the magnitude of its bias current, the directly unmeasurable $\Delta \Phi_{\mathrm{S} / \mathrm{C}} \equiv\left(\Phi_{\mathrm{S} / \mathrm{C}}-\Phi_{p, \mathrm{~S} / \mathrm{C}}\right)$ in (1) can be inferred from measured quantities for use in discriminating trapped spacecraft photoelectrons and in building up the explicit form of the functional sensitivity of the photoemission current to the spacecraft floating potential $\Delta \Phi_{\mathrm{S} / \mathrm{C}}$ implied in (1) with particle inferences of the plasma current collected at that potential.

To summarize, the potential of the spacecraft with respect to the nearby plasma is determined by the balance between the plasma thermal electron current and the spacecraft photoemission. This current balance causes the potential of the spacecraft with respect to the nearby plasma to depend on the plasma current and density. To measure this potential difference between the spacecraft and the nearby plasma, one must have an object at the potential of the nearby plasma. The sphere becomes this object (to within a volt or two) through setting its bias current to a value of typically half of its photoemission current. As the photoemission of the Polar spheres increased from $\sim 100$ to $350 \mathrm{nA}$ with a time constant of $\sim 1$ month, the bias current was adjusted $\sim 20$ times during the first 3 years on orbit, in order to maintain the proper ratio between the two currents. This ratio causes the sphere to float positive with respect to the nearby plasma by a fraction of the e-folding energy of the photoemission spectrum, or by 1 or $2 \mathrm{~V}$.

\section{Spectra and Estimates of the Floating Potential From EFI}

Plate 1 illustrates a period on July 25, 1996, of Polar-Hydra electron data collection at very low kinetic energies, $E_{o b s}$ when there were both real geophysical electrons at low energy and, at times, only photoelectrons contaminating the lower channels. The spectrogram depicts differential energy flux in standard energy time format in three panels. The proxy omnidirectional flux is the average at the given energy over all 12 Hydra sensors regardless of the Sun phase or pitch angle in each $13.8 \mathrm{~s}$; it is the quantity plotted in all three panels.

Plate 1 (bottom) shows the fluxes at their observed kinetic energies, $E^{\prime}$. The bright and variable yellow regions toward the bottom of the energy range are trapped photoelectrons. This impression is confirmed in Plate 1 (middle) where the spectrogram is replotted and two black overlay traces are drawn as suggested by the inferences of the floating potential of the vehicle made from the EFI measurements discussed above. The lower trace is the spin average EFI determination of the floating potential as indicated in the above analysis. The upper trace of the middle panel is biased up $2 \mathrm{~V}$ from that EFI potential. Two points should be made here: (1) the variable yellow patches of the lower panels at lower energies are confirmed to be trapped photoelectrons; (2) the broadcast (lower energy trace) potential does not exclude all bright yellow pop- ulations that appear contiguous in energy, while the biased (upper trace) seems to do a better job at rejecting the photopopulations. The third panel of this figure depicts the omni directional differential energy flux a spacecraft riding at the plasma potential would "see." The differential energy fluxes are plotted at the energies they would have outside the Debye sheath, $E_{D}=E^{\prime}-|e| \Delta \Phi$. This is the energy they would have had were there no accelerating potential and the flux has been corrected consistent with Liouville's theorem. Note the clean and clear photorejection at the lowest energies in Plate 1 (top). The upper trace of the two potential estimates in Plate 1 (middle) was used for the transformations to obtain the upper panel.

Plate 1 also points out clearly the routine types of problems encountered making these low-energy measurements: the volatility and spatial inhomogeneity of the plasma parametrically cause the spacecraft floating potential to vary in a way controlled by the current balance issues discussed above. When there is little plasma electron flux, the potential of the spacecraft can be in excess of $20 \mathrm{~V}$, while tens of minutes later the floating potential is depressed by the arrival of a blast of flux from the cusp around 0730 UT. After comparing a number of such events we have estimated that the EFI spacecraft potential estimate $\Delta \Phi_{\mathrm{S} / \mathrm{C}, \mathrm{EFI}}$ is biased in an absolute sense to be too small compared to what the observed particle spectra would suggest. We have used for the estimate of the spacecraft potential

$$
\Delta \Phi_{\mathrm{S} / \mathrm{C}}=\left|\Delta \Phi_{\mathrm{S} / \mathrm{C}, \mathrm{EFI}}\right|+2 \mathrm{~V} .
$$

For the remainder of this paper when we refer to $\Delta \Phi_{\mathrm{S} / \mathrm{C}}$ we are referring to this positive offset number rather than the modulus of the EFI telemetered potential which is usually plotted in publications as a negative number. There are a variety of possible causes for this offset; among them are the "bias potentials" discussed above.

\section{A More Detailed Introduction to the Physics of the Potential}

In reality, there are several other sources of current than discussed above involved in the balance equation that sets the spacecraft floating potential. Not all the plasma particles that hit the spacecraft "stick," sometimes they eject a secondary that though degraded in energy from the primary, may have sufficient energy to escape the potential well that is primarily sized to trap the low-energy photoelectrons made by solar UV. In addition the ions of the plasma represent a current intercepted by the spacecraft. To the extent that the ions may be hotter than the electrons and when the ambient plasma is at a very low density, the ion contributions to the balance equation are in principle involved, though usually they are smaller than the contributions of the electrons. With all the "puts" and "takes" of the various sources the physics of the determination of the floating potential is still that of current balance.

The spacecraft in sunlight adjusts its potential so that the number of escaping photo-electrons and secondary electrons is just balanced by the ambient net plasma current (caused by electron and ion fluxes) to the surface of the spacecraft. Said differently, the charge on the spacecraft changes in time until the integral of the $\nabla \cdot \mathbf{J}$ over a volume enclosing the spacecraft vanishes. The currents passing through a surface encompassing the entire spacecraft and appendages have at least four causes: 
(1) the flux of photoelectrons that surmount the attractive potential in sunlight that leave the spacecraft vicinity; (2) the incident flux of plasma electrons that come from outside this surface arriving at the spacecraft surface with kinetic energy, $E^{\prime} \simeq E_{\mathrm{obs}} \geq\left|e \Delta \Phi_{\mathrm{S} / \mathrm{C}}\right| ;$ (3) the escaping electrons that result from the ambient plasma electrons impacting the spacecraft surface, usually called the escaping secondary flux; and (4) incident ambient ion flux that is intercepted at the spacecraft, which because of its charge is operationally equivalent to an enhanced secondary loss of electrons. By Gauss' law when the spacecraft reaches an equilibrium potential, the surface integral of all these fluxes must vanish. We proceed to infer the photoelectron flux, $J_{\hbar \nu}$, by determining the net fluxes that compensate for this escaping flux when the vehicle is in equilibrium for a wide range of potentials over the first 10 months of operation of the Polar spacecraft.

As a geometrical aside, it should be clear that the effective area, $A_{J}$, for collecting or emitting fluxes that are caused by the plasma is different than the illuminated area, $A_{S}$, which scales the loss of photoelectrons. Polar's spin vector, $\Omega$, is perpendicular to the plane of its orbit; this causes the angle $\vartheta$ that $\Omega$ makes with the spacecraft sun line to be time dependent. Accordingly, the sunlit area is a seasonal function of time, $A_{S}(t)$, while the plasma current collecting area is time independent and, in subsonic flow, is essentially the geometrical area of the spacecraft. We have approximated the spacecraft as a right circular cylinder of radius $R$ and height $H$, so that its illuminated area, $A_{S}^{*}$, when $\vartheta=\pi / 2$ is

$$
A_{S}^{*}=2 R H \text {. }
$$

The ratio of current collecting to photocurrent emitting area as a function of time is given by

$$
\frac{A_{J}}{A_{S}(t)}=\frac{\pi\left(A_{S}^{*}+2 R^{2}\right)}{A_{S}^{*}|\sin \vartheta|+\pi R^{2}|\cos \vartheta|} .
$$

By determining the plasma return current density, $J_{\mathrm{RC}}$, of ambient ingoing electron fluxes, less secondary outgoing electron fluxes, less incoming ion fluxes and correcting for the above time-dependent ratio of areas we should be able to recover the photoemission current curve as a function of $\Delta \Phi_{\mathrm{S} / \mathrm{C}}$ that should be as independent of time as the solar UV spectrum. Thus current balance is restated as

$$
J_{\hbar \nu}\left(\Delta \Phi_{\mathrm{S} / \mathrm{C}}\right)=\frac{A_{J}}{A_{S}(t)} J_{\mathrm{RC}}\left[\Delta \Phi_{\mathrm{S} / \mathrm{C}}(t)\right],
$$

where $J_{\mathrm{RC}}$ contains all the contributions of types 2-4 enumerated above.

\section{Implementation of Scheme}

By knowing the spacecraft potential $\Delta \Phi_{\mathrm{S} / \mathrm{C}}$ for a range of values when there are plasma measurements, we can determine an experimental calibration curve consisting of ordered pairs $\left(\Delta \Phi_{\mathrm{S} / \mathrm{C}}(t), J_{\hbar \nu}\left(\Delta \Phi_{\mathrm{S} / \mathrm{C}}(t)\right)\right.$, where the return current determined from the Hydra detector is dominated by the incoming electron current density given by the four fold integral over spacecraft skin area elements, $d \mathbf{a}$ (functions of the velocity space polar angles $\theta_{v}$ and $\phi_{v}$ ), the speed variable as well as the accessible solid angles that can reach the spacecraft.

$$
\begin{aligned}
& J_{\mathrm{RC}}[\Delta \Phi(t)]=\frac{|e|}{A_{\mathrm{S} / \mathrm{C}}} \\
& \cdot \iiint \int_{v \Phi}^{\infty} f_{e}\left(\mathbf{v}_{\mathrm{obs}}, t\right) v_{\mathrm{obs}}^{2} \mathbf{v} \cdot d \mathbf{a}\left(\theta_{v}, \phi_{v}\right) \sin \theta_{v} d \theta_{v} d \phi_{v} d v_{\mathrm{obs}} .
\end{aligned}
$$

Equation (7) is schematic in that the limits of integration depend on the Debye length regime of the observations. Care has been taken to perform these integrals with attention to the varying limits of integration that such an approach entails, including solving in each of the 1.6 million spectra the transcendental equations that determine these limits of integration [Whipple et al., 1974]. The lower limit of the speed integration is that minimum speed an electron can have at the spacecraft after traversing the accelerating potential difference across the sheath: $\frac{1}{2} m v_{\phi}^{2}=|e| \Delta \Phi_{\mathrm{S} / \mathrm{C}}$. The solid angle integration is done in a way that averages the flux over different angles of inclination to the spacecraft surface, $d \mathbf{a} \cdot \mathbf{v}$, so that (7) accounts for the incident current density intercepted by the spacecraft surface. In practice, there is a small correction to this current from ejected secondary electrons when the ambient plasma bombards the vehicle. This correction is done in the manner described by Whipple [1981]. The observed secondary current densities are usually a very small correction to the direct electron current density. The ion current to the spacecraft is also involved in the current balance although it is rarely important in sunlight. It has, nevertheless, been computed so that this systematic detail cannot explain any discrepancies indicated below. Since Hydra is an energy per charge detector [Scudder et al., 1995] without mass resolution, determining the ion current assuming all the flux is protons is an upper bound on the true ion current. Given the concerns outlined below we subtract one half of this "proton" current from the other contributions and add in quadrature one half of this ion current as an uncertainty to the overall return current estimate. In some parts of the orbit this may be the dominant source of uncertainty in determining $J_{\mathrm{RC}}$.

If the ambient electron plasma were modeled to be a Maxwellian distribution at temperature $\frac{1}{2} m_{e} w_{e}^{2}=k T_{e}$ the one sided return current density to a plane would have the form

$$
J_{R C}(2 \pi)=\frac{2}{\sqrt{\pi}} n w_{e}\left(1+\frac{v_{\phi}^{2}}{w_{e}^{2}}\right) .
$$

The second term in parentheses a form determines the socalled "focusing" correction term in the long Debye length approximation to the return current of the ambient electrons if the spacecraft were uncharged. The physics of this accelerated contribution to the current is automatically included in the ambient estimates made from the Hydra detector in the form given by (7) as a simple change of variables will demonstrate.

There remain two practical issues for the velocity space integration. For the return current the theoretically required interval is from the lowest speed to the highest that can gain access to the spacecraft from infinity, namely, $\left[v_{\phi}, \infty\right]$. The Hydra particle detectors measure electrons within fixed but programmable energy steps that are roughly logarithmically spaced in a finite range below $20 \mathrm{keV} / q$, while the above integrals require integrals to $\infty$. The second issue is that with fixed energy channels, as the spacecraft floating potential varies there will, in general, not be a sampling of the distribution 
function at the kinetic energy $E(t)$ that corresponds to the neighborhood just above that of the floating potential energy $|e| \Delta \Phi_{\mathrm{S} / \mathrm{C}}$.

The semi-infinite speed interval $\left[v_{\phi}, \infty\right]$ is most easily addressed by transforming the integral with Liouville's theorem to be an integral beyond the Debye sheath by making the energy transformation to Debye velocity space variables

$$
v_{\text {Debye }}^{2}=v_{\text {obs }}^{2}-v_{\phi}^{2},
$$

with limits of integration $[0, \infty]$. By a further transformation this semi-infinite interval may be mapped onto the finite interval $\chi=[0,1]$ with

$$
\chi\left(v_{\text {Debye }}\right)=\frac{v_{\text {Debye }}}{a+v_{\text {Debye }}} .
$$

The constant $a$ of the transformation is chosen so that

$$
\chi\left(v_{\text {Debye }} \equiv\left\langle v_{\text {Debye }}^{2}\right\rangle^{1 / 2}\right)=\frac{1}{2}
$$

This practice allows the inclusion of $f(\chi=1) \equiv 0$ as a point in a finite domain of the numerical integration while leaving room in the interior of $[0,1]$ for the structure of the integrand determined from the measured distribution function. It is assumed that the rate of convergence of $f$ to zero is sufficiently rapid to overpower the divergent Jacobian and the finite powers of $v$ in the integrand so that the integrand's derivative goes to zero as $\chi \rightarrow 1$. In the routine processing we keep track of the percentage contribution to the plasma return integral made by the last interval $\left[\chi_{*}, 1\right]$, where $v_{\text {Debye }}\left(\chi_{*}\right)$ is the last speed where finite counts were observed. This fraction is referred to below as the "black" fraction; it will be shown to be an important indicator of circumstances when Hydra is surveying the dominant energy range of the returning plasma current, $J_{\mathrm{RC}}$ This is the circumstance when the statistical empirically constructed photoemission curve can be used to infer the floating potential when it is not provided by the EFI measurements.

The remaining difficulty in determining the return current value for a given spectrum concerns the behavior of $f_{e}\left(v_{\text {obs }} \rightarrow\right.$ $\left.v_{\phi}\right)$. In practice, there are usually no measurements precisely at $f_{e}\left(v_{\phi}\right)$, so the trend of $f_{e}(v)$ for $v_{\text {obs }}>v_{\phi}$ must be used to infer the behavior down to the lowest positive energy required. Because the electrons are invariably ultrasubsonic there are measurements in all $4 \pi$ direction that approach the origin in $\mathbf{v}_{\text {Debye }}$ space. Thinking of the phase space before encountering the spacecraft sheath, the unmeasured part of $f_{e}\left(\mathbf{v}_{\text {Debye }}\right)$ is a small volume near the origin in the Debye variables. Our procedure is to assume that $\ln f_{e}\left(\mathbf{v}_{\text {Debye }}\right)$ has a second-order Taylor series in three variables $\left.\left(v_{x}, v_{y}, v_{z}\right)\right|_{\text {Debye }}$ and to then determine the 10 unknown coefficients in the neighborhood of the origin as constrained by the data that are measured at adjacent, but higher energies. It is this routine interpolation of $f(\mathbf{v})$ at the lowest energies that is the basis of the moments as full numerical 3-D integrations as well as the routine return current determinations.

\section{Results of the Routine Determination of $J_{\hbar \nu}(t)$}

Plate 2 illustrates the frequency of occurrence of the above ordered pairs $\left(\Delta \Phi_{\mathrm{S} / \mathrm{C}}, J_{\hbar \nu}\right)$ for the ten months of data from the beginning of the mission when floating potential estimates from EFI are available after screening them for their integrity with sub-spin samples and removing time intervals of shadows, or EFI self calibrations. To simplify the analysis intervals when the PSI plasma neutralizer [Moore et al., 1995] was running were excluded. This plot reveals a well-developed curvilinear boundary from top left-hand corner to bottom right-hand corner that is a candidate for the photoemission calibration curve that we seek. As shown below over $60 \%$ of the 1.6 million spectra examined in this 10 month period are contained within the immediate vicinity of this bounding ridge. In addition to the scattered points near the curve, there are two notable clusters below the upper bounding curve: (1) at low potentials, 2-4 V, in the bottom left-hand corner there are frequent departures well below the bounding curve that represent several orders of magnitude shortfall in Hydra's direct determination of the return currents and hence $J_{\hbar \nu}$ plotted in Plate 2; and (2) points between 15 and $30 \mathrm{~V}$ with $J_{\hbar \nu}$ shortfalls that are generally smaller than those in group 1 just discussed.

The departures of points from the bounding curve vary from week to week and month to month in a way that will be discussed elsewhere (X. Cao et al., Properties of very cold ( $T_{e} \simeq 0.1 \mathrm{eV}$ ) electrons within the magnetosphere, submitted to Journal of Geophysical Research, 1999, hereinafter referred to as submitted manuscript, 1999). The precise frequency of occurrence in each region reflects the intersection of Polar's orbit with regions where cold plasma may be surmised to be present. We will return in the discussion section to these exceptions from the single valued bounding curve that is the focus of this paper. For reference and for synthesis of the present work Plate 2 also contains curves that are the predictions of three model fits of the photoelectric current calibration curve: (1) the dotted curve illustrates a previous photoemission current density curve [Pedersen, 1995]; (2) the dashed curve is a determination of the boundary discussed later in this paper in Figure 1; and (3) the solid line is the best fit deduced from Polar data in this paper in Figure 2. We now proceed to describe the empirical process for finding this bounding curve in a methodical way.

Two approaches suggest themselves: devise by sorting methods a procedure for highlighting the bounding curve as an important curve to compare with previous work for $J_{\hbar \nu}$. After obtaining the fit to this empirical curve (1) look at what distinguishes the data points not close to this limiting curve or (2) ascertain the statistical properties of those data not near the bounding curve and then fit the remainder to define the bounding curve.

In Plate 3 we illustrate the results of the first approach. All the data were binned in contiguous potential bins commensurate with the stated precision of the $\Delta \Phi_{\mathrm{S} / \mathrm{C}}$ measurement. The mode of $J_{\hbar \nu}$ in each potential bin is determined from the low "black fraction" current densities in each potential bin. The variance of this modal assignment has been estimated from the points in the potential bucket that are above the modal value assuming there is a mirror set of points below the mode. A best fit model for the bounding curve of the function class used by Pedersen [1995]; Escoubet et al. [1997] has been found. The modal points and their variances together with the best fit curve and fit parameters and their errors based on the analysis of the Hessian [Press et al., 1992] at the optimal fit parameters are indicated in Figure 1. Overlays of predictions from previous work [Pedersen, 1995; Escoubet et al., 1997] in Figure 1 confirm the likelihood that this upper boundary is determined by the photoemission curve that we seek to define. Better agreement exists between the present more extensive data set and the earlier work of Pedersen [1995]. Differences do remain, 


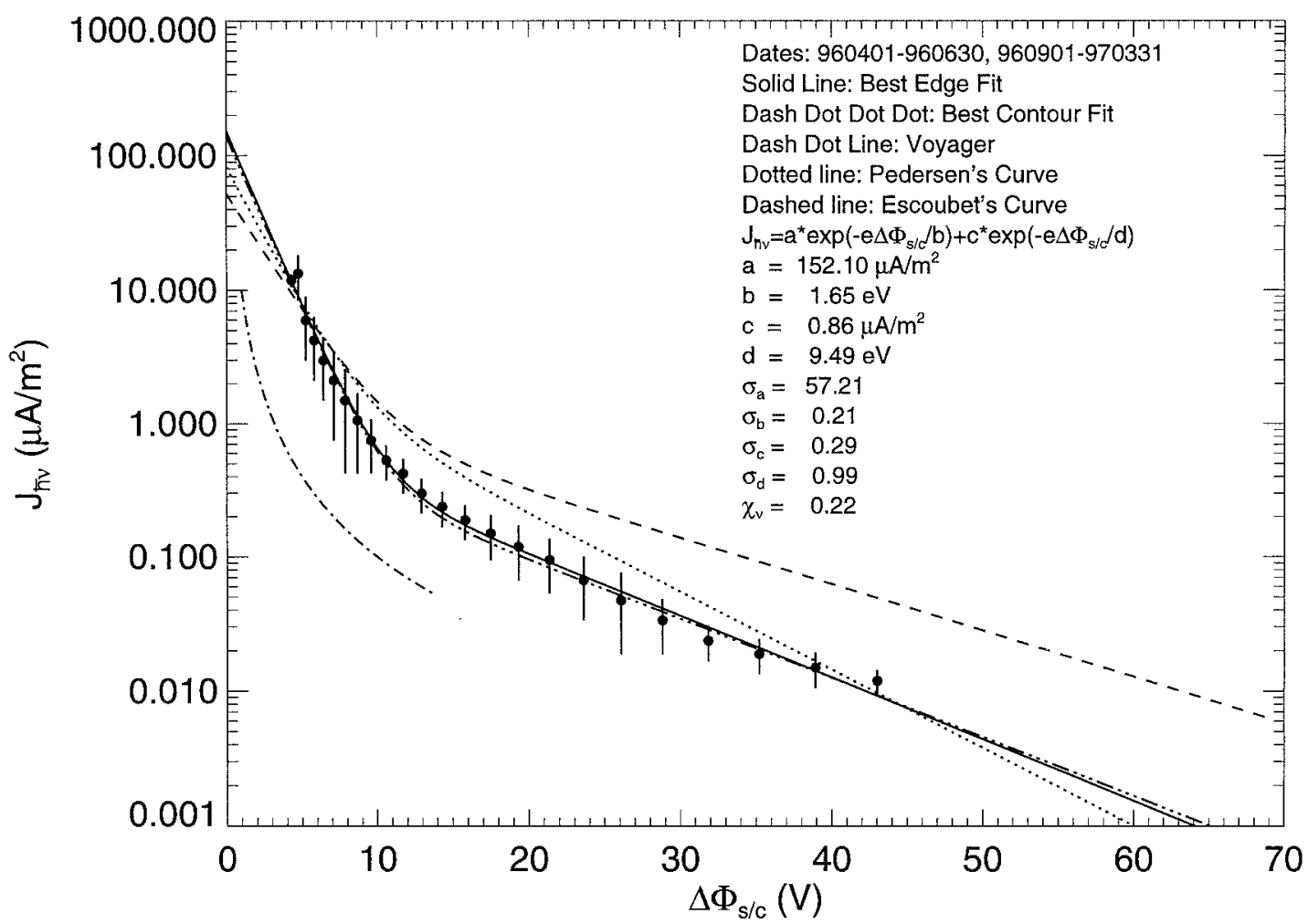

Figure 1. Determine bounding curve by characterizing the precipitous edge.

however, especially in the form of this curve with increasing floating potential. Possible reasons for these differences are discussed below.

Alternatively, the data have been normalized in each potential bin with probabilities $P_{i, j}^{\Phi}$ such that the sum of the probabilities in the same $\Phi$ bin is unity. We then construct the analogous normalized probability $P_{i, j}^{J}$, where the data are organized by their current densities. Each subscript pair $i, j$ of each probability refers to the same location in $\Phi, J_{\hbar \nu}$ space. We form the composite probability $C_{i, j}=P_{i, j}^{\Phi} P_{i, j}^{J}$ to give an indication where the joint correlation is enhanced. Using this composite probability curve to define the bounding curve we can then look for the outliers against it. Rank ordering this joint probability and then encircling the location of the highest $\mathrm{Q} \%$ of the probability allows the extraction of the desired ridge. The results of such a procedure are illustrated in Figure 2 , where the last bounding contour encompasses $Q=60 \%$ of the 1.6 million spectra examined. Returning to the potential bins, we determine the mean and variance of the points that are simultaneously within the potential bin and within this outer contour. We have fit these data to the same model form as used by Pedersen [1995] and Escoubet et al. [1997]. All three curves are compared in Figure 2. The fit parameters on Figure 2 are those of the new binning procedure of this paragraph.

Finally, we have surveyed the $\left(\Delta \Phi_{\mathrm{S} / \mathrm{C}}, J_{\hbar \nu}\right)$ scatter plot looking for systematic effects that segregate the data. Plate 4 is a "black fraction" indexed survey that was particularly revealing. The black fraction is generally low nearer Pedersen's and our $60 \%$ cumulative probability ridge curve than further away. Operationally, considering only $J_{\hbar \nu}$ estimates that are sufficiently convergent (for example, "black fraction" $<3 \times 10^{-3}$ ) would appear to segregate points near to the bounding spine of the probability curve. For future usefulness of this statistical relation discussed below, it is important to be able to anticipate when all the current to the spacecraft has been inventoried from the measured Hydra data alone. When the black fraction is high this signals one of two possibilities: there is a lot of current either above or below the sensitivity range of Hydra. If there is substantial missing current below Hydra's energy range, this reduces the real fraction of the current inferred above its threshold. A secondary discriminator between these two possibilities is the energy location of the peak electron flux. If this peak is inferred to be above Hydra's energy range, this is another way that the operational value of black could be high. In either case such points are not places where Hydra data plus the statistical relation of equation (10) can be used in a bootstrap fashion to find the floating potential $\Delta \Phi_{\mathrm{S} / \mathrm{C}}$. Conversely, these are the input data points that should be excluded from a statistical determination of the photoemission potential relation.

Using the above approaches to enhance the bounding upper ridge of the photoemission scatterplot, we have fit the selected points to models formed as the sum of two Gaussian distributions.

$J_{\hbar \nu}\left(\mu A / m^{2}\right)=a \exp \left(-\frac{e \Delta \Phi}{b}\right)+c \exp \left(-\frac{e \Delta \Phi}{d}\right)$.

Such a model form is suggested since this current curve as a function of $\Delta \Phi$ reflects the distribution of photocurrent ejected from a metal in sun light, where there is a characteristic UV temperature of the Sun as well as far UV enhancements that are well known. Crudely this model reflects these temperatures in the $b$ and $d$ fit parameters. As may be seen in Table $1(1.4<$ $b<2 \mathrm{eV}$ and $8.5<d<11 \mathrm{eV})$, respectively, are in line with 

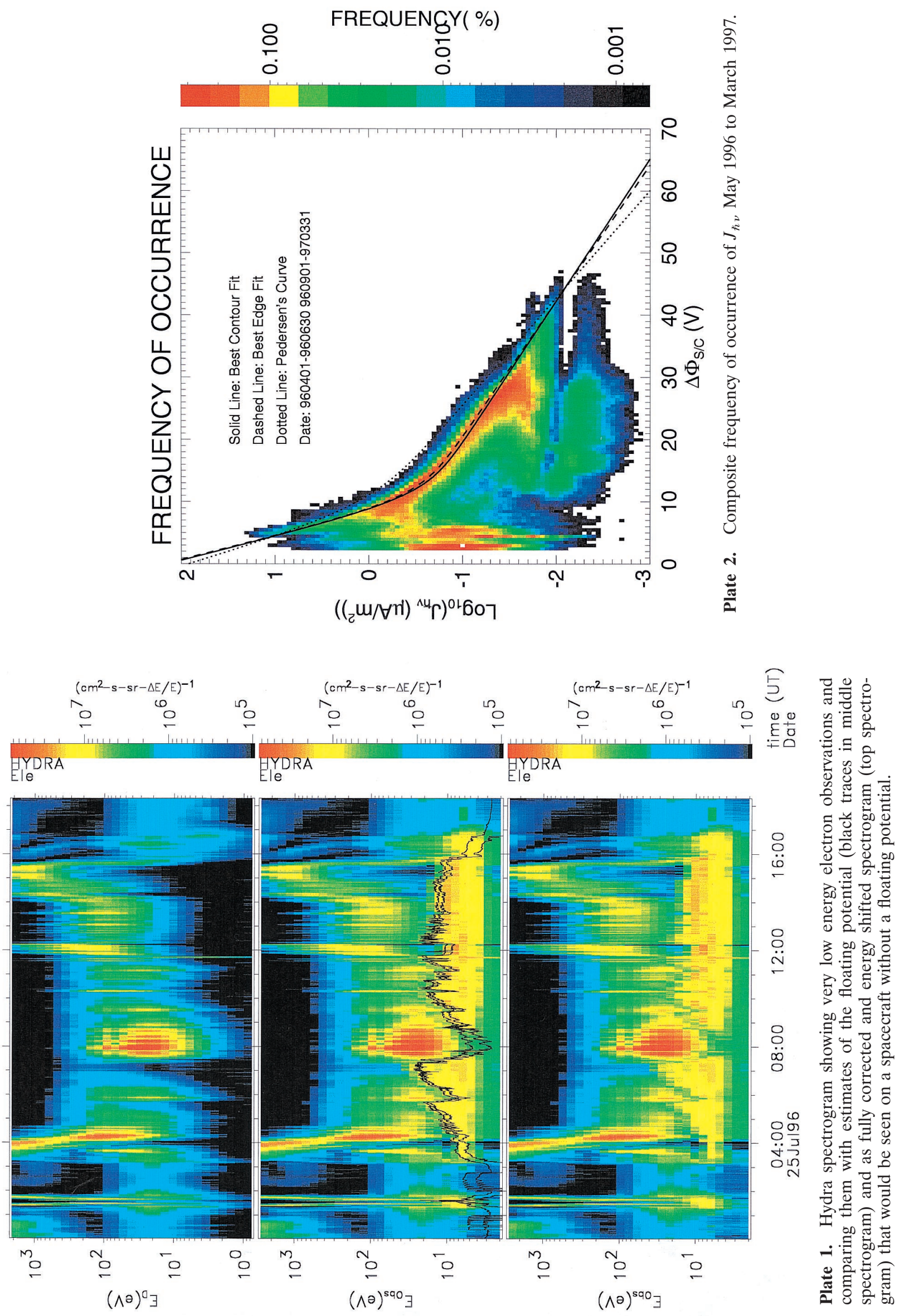

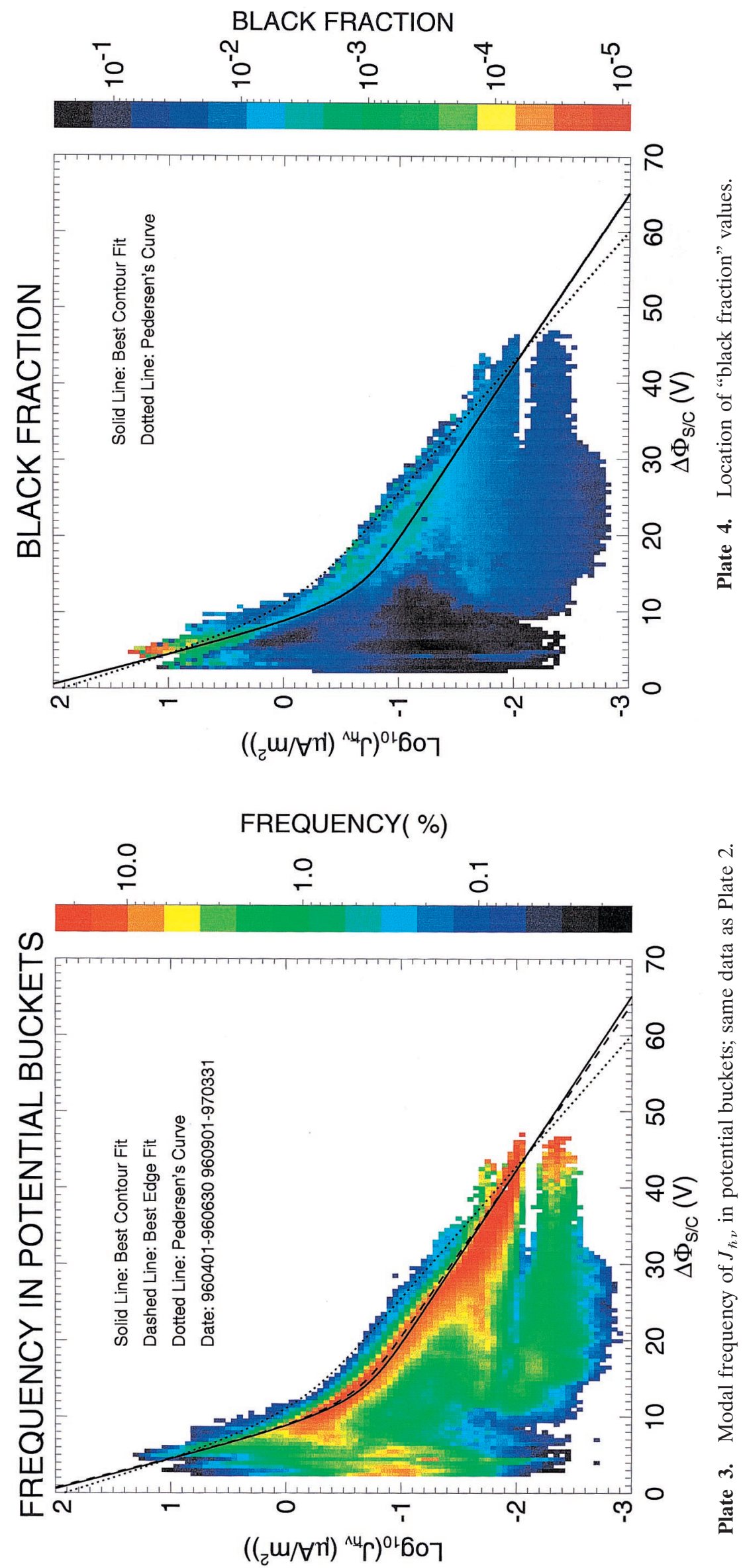


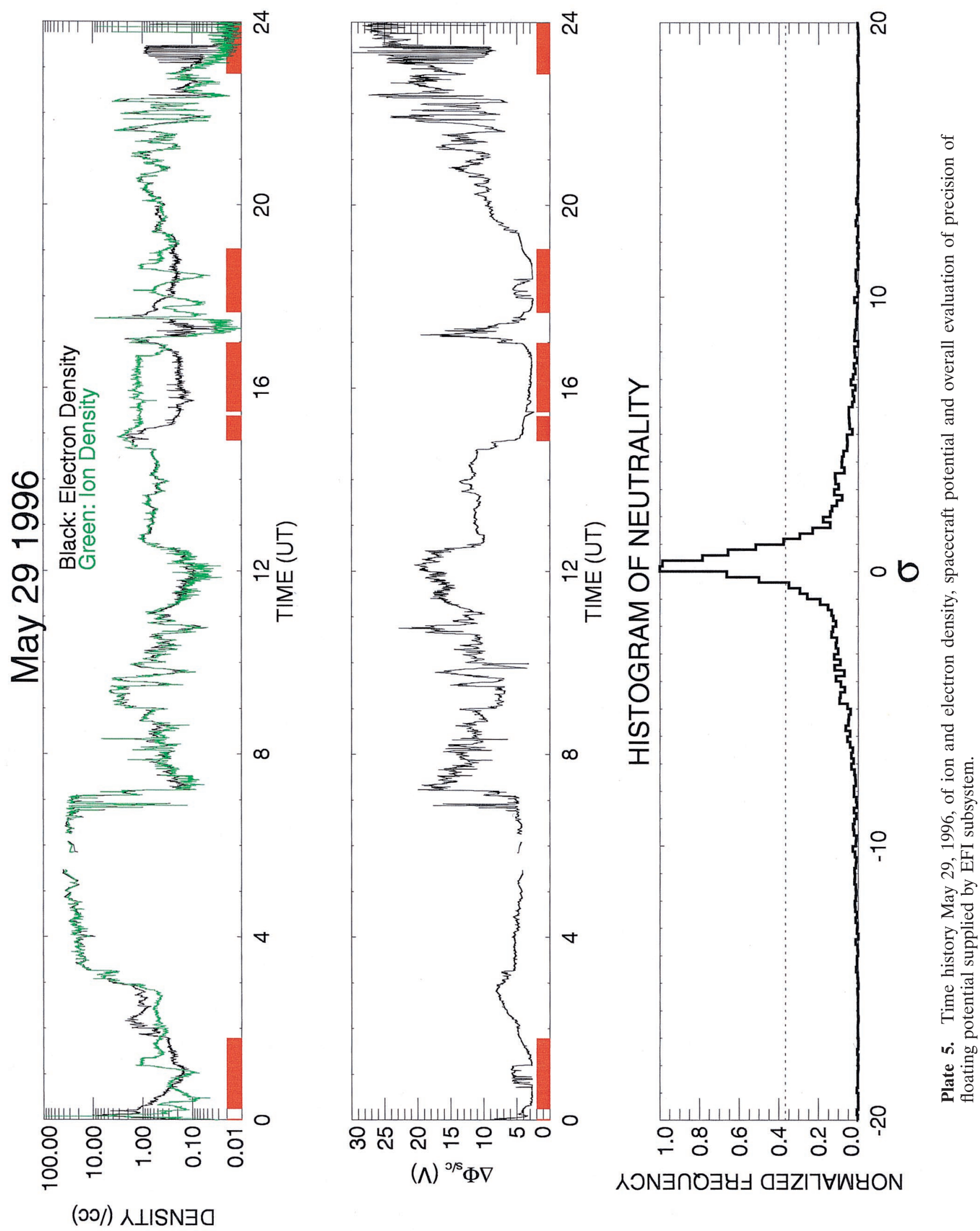




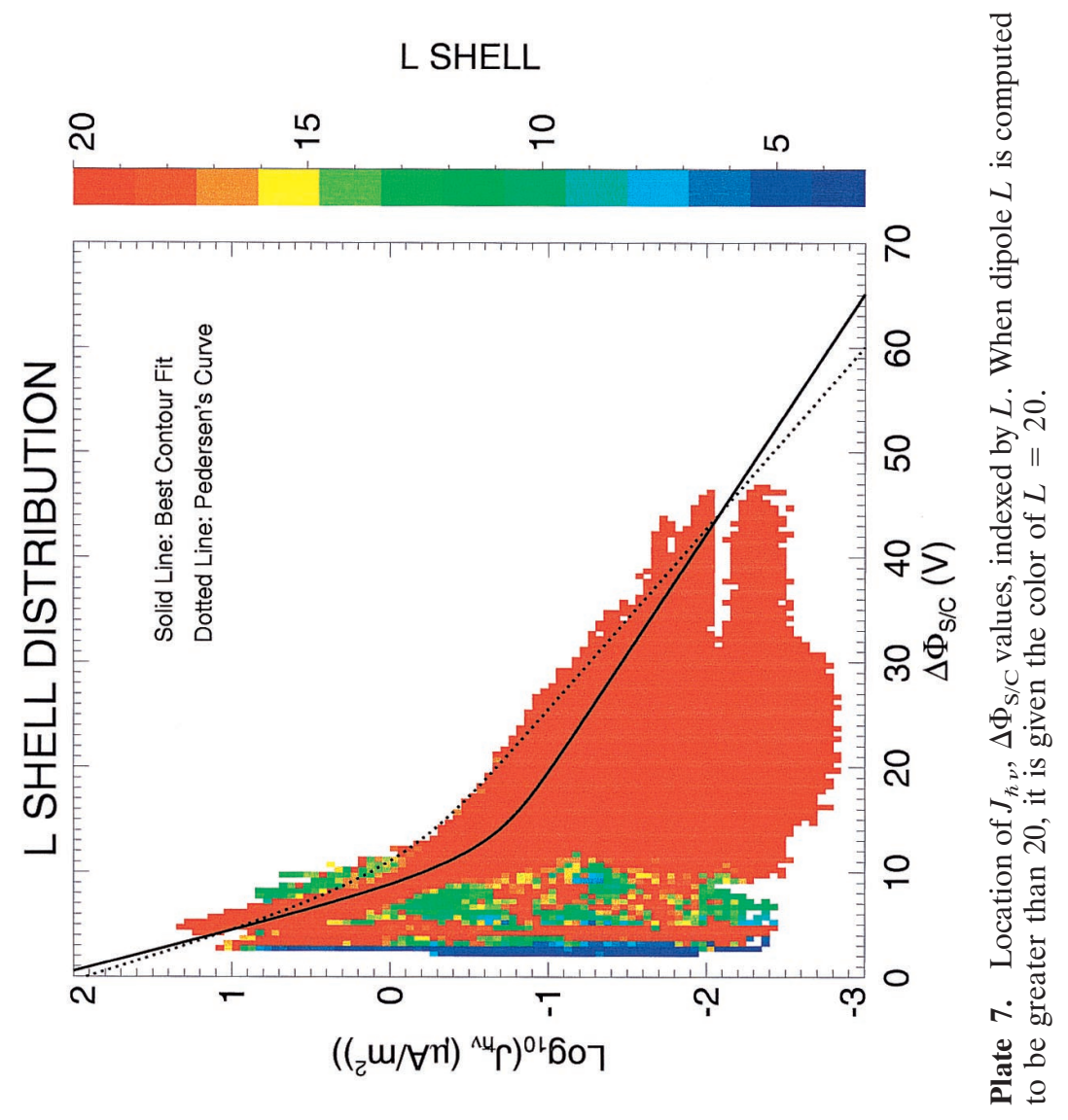

RADIUS

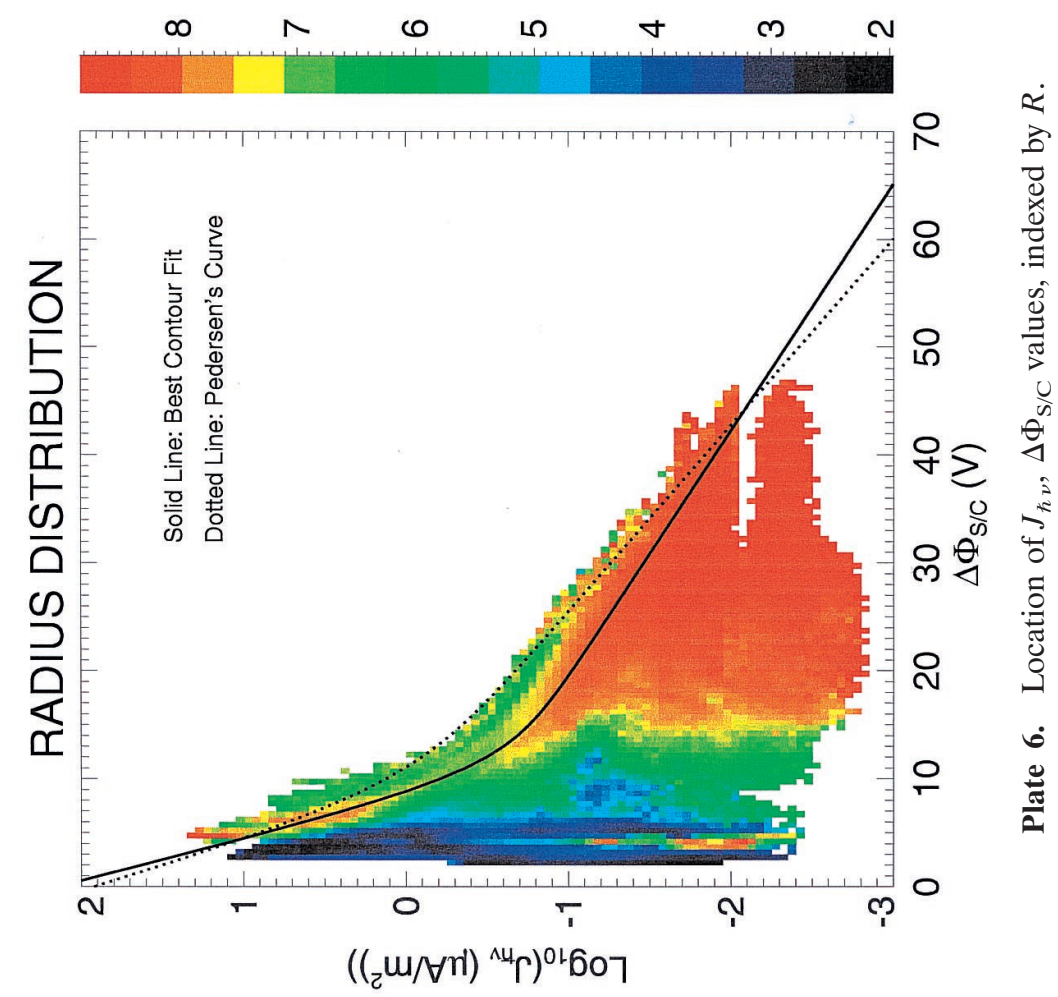




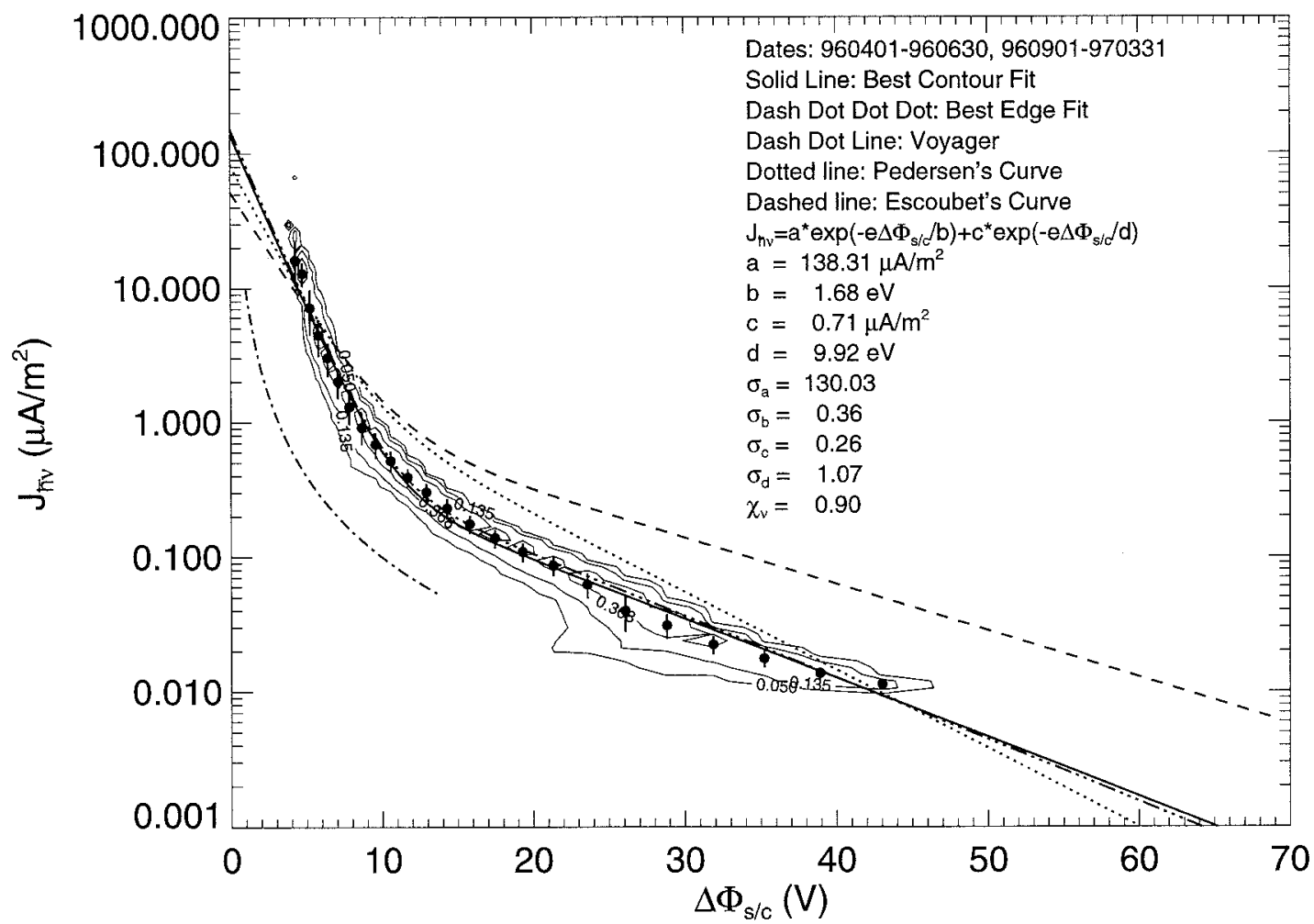

Figure 2. Double Gaussian characterization of the location of high joint probability readings.

such a simple picture of a very complex array of lines in this region of the ultraviolet spectrum that are effective in ejecting photoelectrons. Such a functional form was used previously by Pedersen [1995] modeling ISEE and GEOS data and more recently by Escoubet et al. [1997]. In Table 1 we present a comparison of the fit parameters by various approaches and a comparison with those found previously with ISEE, GEOS, and Voyager. In Table 1, the comparisons between present and previous work should concentrate on the keyed entries. Because the Escoubet et al. [1997] data set is a superset of Pedersen [1995], the variation between these two rows indicates, if only approximately, the uncertainties in their fit parameters. Of interest is the average photocurrent that these relations would suggest was emanating from the spacecraft if there where no potential barrier, $J_{\hbar \nu}\left(\Delta \Phi_{\mathrm{S} / \mathrm{C}}=0\right)$. Within the function class used to model this relation $J_{\hbar \nu}\left(\Delta \Phi_{\mathrm{S} / \mathrm{C}}=0\right)=a$ with $a=152 \pm 57 \mu \mathrm{A} / \mathrm{m}^{2}$. Considerable caution must be taken in using this number as it represents an extrapolation of the observations to the desired zero potential regime. The large uncertainty there is typical of steeply rising curves such as the cold exponential. Clearly, the absolute size of this number is model dependent. Its precision could only be improved by a more detailed functional model of the expected photoemission current, as a convolution of the UV spectrum with the materials property of the spacecraft.

The Pedersen and Escoubet studies estimate the plasma part of the current using reported densities and temperatures in formulae like (8) for the current as if the various plasma regime distribution functions were Maxwell Boltzmann distributions. By contrast the present study has done the more fundamental integrals in (7) over the observed velocity distribution as a function of energy and angle, thereby removing one

Table 1. Comparison of Fit Parameters by Various Approaches and a Comparison With Those Found Previously With ISEE, GEOS, and Voyager

\begin{tabular}{|c|c|c|c|c|c|c|c|c|c|}
\hline & \multicolumn{8}{|c|}{ Source } & \multirow[b]{2}{*}{$\chi_{v}^{2}$} \\
\hline & $a$ & $\pm \sigma_{a}$ & $b$ & $\pm \sigma_{b}$ & $c$ & $\pm \sigma_{c}$ & $d$ & $\pm \sigma_{d}$ & \\
\hline Units & $\mu \mathrm{A} / \mathrm{m}^{2}$ & $\mu \mathrm{A} / \mathrm{m}^{2}$ & $\mathrm{eV}$ & $\mathrm{eV}$ & $\mu \mathrm{A} / \mathrm{m}^{2}$ & $\mu \mathrm{A} / \mathrm{m}^{2}$ & $\mathrm{eV}$ & $\mathrm{eV}$ & $\cdots$ \\
\hline Joint probability & 138 & 130 & 1.68 & 0.36 & 0.71 & 0.26 & 9.92 & 1.07 & 0.9 \\
\hline Edge fit & 152 & 57 & 1.65 & 0.21 & 0.86 & 0.29 & 9.49 & 0.99 & 0.22 \\
\hline Pedersen & $80^{*}$ & $\cdots$ & 2 & $\cdots$ & $3^{*}$ & $\cdots$ & 7.5 & $\cdots$ & $\cdots$ \\
\hline Escoubet et al. & $50.4^{*}$ & $\cdots$ & 2.40 & $\ldots$ & $1.49^{*}$ & $\ldots$ & 12.63 & $\ldots$ & $\ldots$ \\
\hline Joint probability $\dagger$ & $42 \dagger$ & $40 \dagger$ & 1.68 & 0.36 & $0.58 \dagger$ & $0.21 \dagger$ & 9.92 & 1.07 & $\cdots$ \\
\hline Edge fit $\dagger$ & $45 \dagger$ & $17 \dagger$ & 1.65 & 0.21 & $0.70 \dagger$ & 0.23 & 9.49 & 0.99 & $\cdots$ \\
\hline
\end{tabular}

*Estimated at the bias sphere of the electric instruments, while estimates of amplitudes $(\mathrm{a}, \mathrm{c})$ are made at the spacecraft surface of Polar. $\dagger$ Amplitudes reflecting removal of the $2 \mathrm{~V}$ offset in the Polar spacecraft amplitudes using the exponential shift and the cold and hot fit "temperatures" (b, d) without correcting the 1.6 million four-dimensional integrals. 


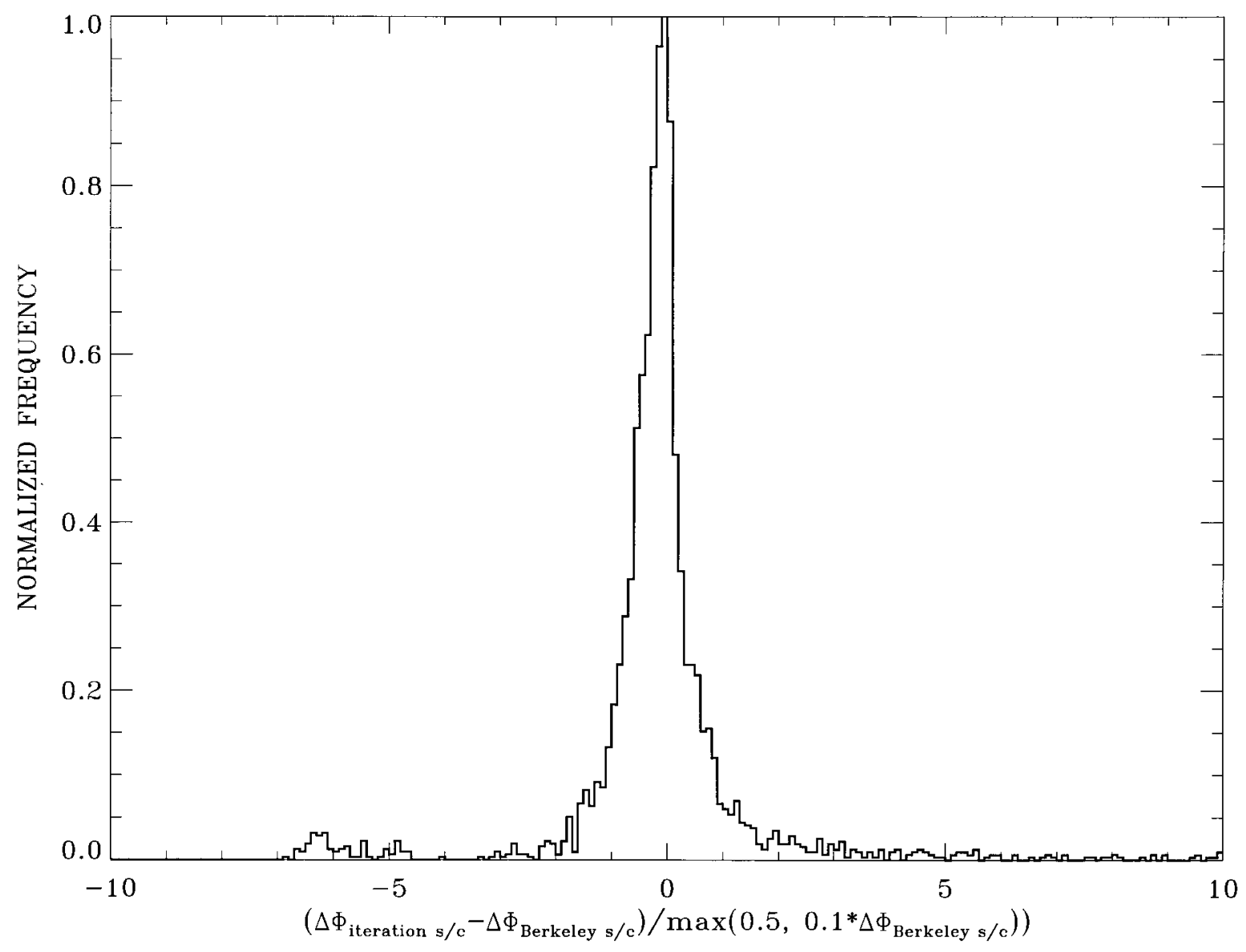

Figure 3. Demonstration of the successful bootstrap iteration strategy for recovery of the floating potential only using the statistical photoemission current potential relation and the Hydra data on May 29, 1996.

possible source of error in obtaining the underlying, and supposedly slowly varying photoemission current floating potential relation we seek. This difference is a possible source of the different trends of the current with floating potential seen in the present study versus those of Pedersen and Escoubet. In addition, the present study includes many more independent measurements than either of the Pedersen or Escoubet surveys.

The "edge fit" entry is considered the best representation of the present Hydra-EFI effort to characterize the photoemission potential relation. The Voyager PLS determination [Scudder et al., 1981] of the return current relationship yielded a relationship $J_{\hbar \nu}\left(\mu \mathrm{A} / \mathrm{m}^{2}\right) \simeq 10 \Delta \Phi_{\mathrm{S} / \mathrm{C}}^{-2}$ for potentials between 1 and $12 \mathrm{~V}$ that has the same trend but a different absolute level as the above synthesis and has been overlaid on Figures 1 and 2 with the dash-dot-dash curve to show its relationship.

\section{Assessment of the Method}

As a measure of the success of this synthesis and as a test of our ability to use it to infer the floating potential when EFI cannot provide such an estimate, we have reprocessed a month of Hydra data using only the average photoemission current curve as our external calibration. For each spectrum we first assume a first iterate for the potential, $\Delta \Phi^{(1)}$, and then determine from the Hydra data the corresponding return current, $J_{\hbar \nu}^{(1)}\left(\Delta \Phi_{\mathrm{S} / \mathrm{C}}^{(1)}\right)$, parametric in that assumption. We then inquire if the coordinate pair $\left(\Delta \Phi^{(1)}, J_{\hbar \nu}^{(1)}\right)$ is consistent with the statis- tically characterized calibration curve. It usually is not, and an iterative procedure is used to converge on a potential $\Delta \Phi_{\mathrm{S} / \mathrm{C}}^{*}$, so that the associated $J_{\mathrm{RC}}^{*}\left(\Delta \Phi_{\mathrm{S} / \mathrm{C}}^{*}\right)$ is consistent with the statistical photoemission curve as modified for the local epoch through the geometrical ratio of areas. (In production the first iterate is assumed to be the last convergent value if it is not too far away in time from the present spectrum.) Figure 3 illustrates the occurrence frequency of this difference distribution between the EFI spacecraft potentials, $\Delta \Phi_{\mathrm{EFI}}$, and those inferred by Hydra data alone by iterating against the statistical photoemission curve (that is now de facto a calibration curve), $\Delta \Phi_{\text {Hydra }}$, normalized by the expected measurement error. The histogram clearly demonstrates that the potential is recovered on average correctly and that the dispersion, $\simeq 1$, between the iterated potential and the measured potential is well within the estimated error.

To assess the precision of the floating potential assignment, $\Delta \Phi_{\mathrm{S} / \mathrm{C}}$, we now compare the electron and ion number densities determined by numerical integration that corrects for $\Delta \Phi_{\mathrm{S} / \mathrm{C}}$ provided by EFI and inquire what additional change in the potential would be required mathematically to enforce precise neutrality. The required correction $\delta \Phi_{\mathrm{S} / \mathrm{C}}$ is approximately determined by

$$
\delta \Phi_{\mathrm{S} / \mathrm{C}}=-k T_{e} \ln \left[\frac{n_{e}}{n_{i}}\right] .
$$

We plot in Plate 5 the frequency distribution of the dimensionless quantity 


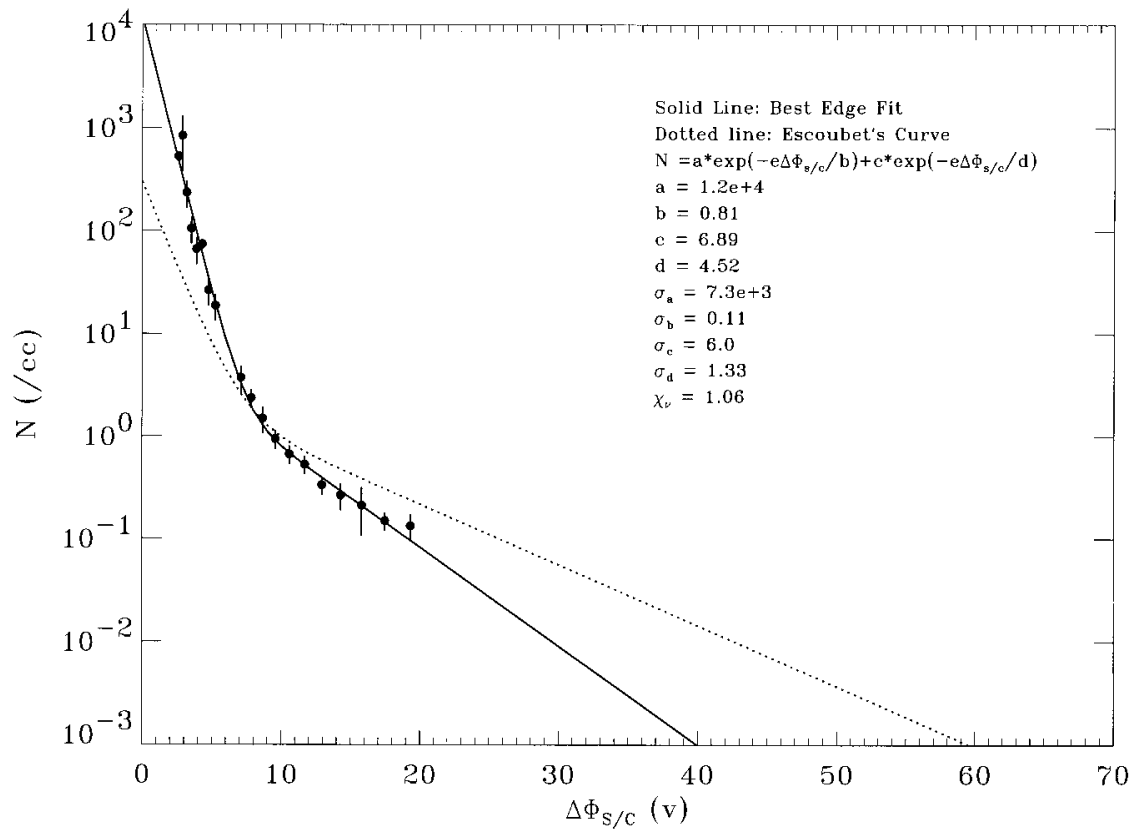

Figure 4. Empirical inventory of electron densities and potentials on Polar from Hydra, PWI and EFI for May 29, 1996. The UHR density estimates (those above the density of 50/cc) form a smooth extension of the density curve densities for Hydra points within the high probability contours of the $J_{\hbar \nu}$ statistical curve. The overlaid curve from Escoubet et al. [1997] has been shifted by 2 V, since its fit coefficients were established in terms of the probe potential, not that of the spacecraft potential as has been done for the Hydra-EFI-Polar construction. In this way the Polar curve and that from ISEE are directly comparable.

$$
\sigma=-\frac{k T_{e}}{e \Delta \Phi_{\text {error }}} \ln \left[\frac{n_{e}}{n_{i}}\right],
$$

where

$$
\begin{aligned}
\Delta \Phi_{\text {error }}= & \left\{\left[\max \left(0.5 \mathrm{~V}, 0.1 \Delta \Phi_{\mathrm{S} / \mathrm{C}}\right)\right]^{2}\right. \\
& \left.+\left[k T_{e} \sqrt{\left(\left(\frac{\delta n_{e}}{n_{e}}\right)^{2}+\left(\frac{\delta n_{i}}{n_{i}}\right)^{2}\right)}\right]^{2}\right\}^{1 / 2}
\end{aligned}
$$

is the expected error for the reconstruction. The constructed frequency of occurrence distribution of $\sigma$ has nearly vanishing mean value, and half width at full maximum that is essentially at the unit width expected if errors have been estimated correctly. The top two panels of Plate 5 illustrate the time evolution of the computed electron (black) and ion (green) densities, and the variation of the $\Delta \Phi_{\mathrm{S} / \mathrm{C}}(t)$ for the period of the histogram just discussed. Red bars highlight regions in the nominal plasmasphere, where the inversion calibration against the potential is not expected to work well as discussed in the next section. These points were omitted based on orbital location in the histogram in the Plate 5 (bottom).

Another qualitative expectation of probe theory as argued by Pedersen [1995], and illustrated by Escoubet et al. [1997], is that the density is nearly a single valued function of the spacecraft potential, being higher when $\Delta \Phi_{\mathrm{S} / \mathrm{C}}$ is lower. This is illustrated with the present data set for one day that contains a full orbit in Figure 4. The UHR points at very low potentials for densities in excess of $50 \mathrm{~cm}^{-3}$ are provided by the PWI team (J. D. Menietti, private communication, 1999). The remaining density points are directly from numerical integration of the Hydra data corrected for the potential when the "black fraction" is acceptable. Figure 4 shows the clear progression from Hydra densities and the potentials sizeable, down to low po- tentials and very high densities typical of the plasmasphere, accompanied by further decreases in the potential, that still remain positive. While the spacecraft remains at a positive potential relative to the plasma, the Polar curve contains higher densities than does the curve of Escoubet et al. [1997] that is shown for comparison. The Escoubet curve does not attempt to correct for the bias voltage of the spherical probe which is the probable cause for this apparent disagreement ( $\mathrm{P}$. Escoubet, private communication, 1999). Since the UHR determinations of density are rather precise (involving determinations of frequencies measured by PWI and corrections that involve the mean magnetic field) the ordinate of these points cannot be suspect. This composite PWI UHR curve of densities joins smoothly onto a curve determined from the direct numerical integration of counting individual particles, correcting for energy shifts, etc. with a different collection of systematic corrections.

We have performed a model fit to a two-component Gaussian curve with fit parameters indicated on Figure 4. Because the Polar spacecraft has a seasonally varying illuminated area, this curve is seasonally dependent and is not therefore a universal curve for this spacecraft independent of time. We will present further statistical data addressing the point in future work. For the present the continuity of this curve between the UHR points from PWI (that are determined independent of the knowledge of the floating potential) and those from Hydra shows the integrity of the $2 \mathrm{~V}$ shift argued in the introduction on the basis of the editing of photoelectrons from the electron energy spectrum.

\section{Outliers}

As a clue to the cause for the systematic group of outliers below the smooth bounding photoemission curve that we have 
determined in this paper illustrated in Plate 2, we plot in Plates 6 and 7 the average occurrence location of all the points contained in Plate 2 but now color coded by the $(R, L)$ coordinate of the observation. From these figures it is clear that the typical points in the "low $\Delta \Phi_{\mathrm{S} / \mathrm{C}}$ " anomalous parts of the $J_{p e}, \Delta \Phi_{\mathrm{S} / \mathrm{C}}$ space of Plate 2 are localized inside the nominal plasma sphere. The other group of outliers from the high probability curve of Plate 2 at higher potentials are from higher $L$ (Plate 7) and in the polar cap over a range of radial locations.

In a related paper (X. Cao et al., submitted manuscript, 1999) we illustrate that these regions are places where dense and cold electrons are found that make up the "missing" plasma return current as estimated by their distance below the statistically defined calibration curve. From the systematic location of these points it is clearly nearly impossible to infer the potential if it is not available within the plasmasphere. Fortunately, when the spacecraft is in this region there is usually a high density and low floating potential, making the assumption of a minimal potential not too controversial, except for its use in inferring a precise plasmaspheric electron temperature.

\section{Summary}

A method of determining the average photoemission current as a function of the floating potential of the GGS-Polar spacecraft has been implemented and evaluated. While the steady average photoemission curve can be reproducibly recovered, and can be used to infer the spacecraft floating potential, intervals that do not fit this simple pattern suggest the presence of time varying, but geophysically organized populations of dense, cold electrons below Hydra's $5 \mathrm{eV}$ low-energy threshold. Routine determinations of the floating potential by iteration against this return current calibration have been shown to recover the measured potentials used to generate the return current curve and to permit quantitative analysis of plasma measurements when the onboard sensors of the spacecraft potential are inoperative. This capability is especially important for traversals of the polar cusp when the EFI sensors can oscillate and be unable by themselves to provide estimates of Polar's floating potential.

We have also validated the quantitative precision of the spacecraft floating potential indirectly for the ten months of data by producing a time invariant $J_{\hbar \nu}$ curve and also by showing the precision of tests of quasi-neutrality from the direct quadratures of the oppositely energy shifted ion and electron distributions.

The plasma electron current depends on the ambient distribution function of the electrons primarily and the spacecraft potential via (7). This in turn implies an interrelationship between the density, the electron temperature, and the electron focusing factor, which depends on the floating potential. Pedersen [1995] and Escoubet et al. [1997] have argued that the temperature dependences are sufficiently weak as to allow the density to be the primary determinant of the actual floating potential achieved. An example of quasi-independence has been illustrated (Figure 4) for one day in the life of Polar. Because Polar's attitude is seasonally dependent, the exhibited density potential curve is not time invariant.

Acknowledgments. The present results of the HYDRA investigation were made possible by the decade-long hardware efforts of groups led at NASA-GSFC by Keith Ogilvie, at UNH by Roy Torbert, at MPAE by Axel Korth and at UCSD by W. Fillius. Discussions with and manuscript comments by C. A. Kletzing are gratefully acknowledged. Discussions with Arne Pedersen and Elden Whipple were also helpful in aspects of this work. We thank D. A. Gurnett for access to the UHR resonance data for Figure 9. At Iowa we would like to acknowledge the cooperative software support of R. D. Holdaway and J. B. Faden. The HYDRA portion of this research at Iowa was performed under NASA Grant number NAG52231 and the German support for Hydra under DARA grant 50 0C 89110 . The EFI participation in this work is supported under NASA grant NAG5-3182. The UHR digitization was supported under NASA Marshall subcontract NAG8-1153 to the University of Iowa.

Hiroshi Matsumoto thanks P. J. Kellogg and another referee for their assistance in evaluating this paper.

\section{References}

Bame, S. J., J. R. Asbridge, H. Felthauser, J. Glore, G. Paschmann, P. Hemmerich, K. Lehman, and H. Rosenbauer, ISEE-1 and ISEE-2 Fast Plasma Experiment and the ISEE-1 Solar Wind Experiment, IEEE Trans. Geosci. Electron., GE-16, 216-220, 1978.

Escoubet, C. P., A. Pedersen, R. Schmidt, and P. Lindqvist, Density in the magnetosphere inferred from the ISEE-1 spacecraft potential, $J$. Geophys. Res., 102, 17,595-17,609, 1997.

Feldman, W. C., J. R. Asbridge, S. J. Bame, M. D. Montgomery, and S. P. Gary, Solar wind electrons, J. Geophys. Res., 80, 4181-4196, 1975.

Harvey, P., et al., The GGS/Polar magnetic fields investigation, in The Global Geospace Mission, edited by C. Russell, pp. 583-596, Kluwer Acad., Norwell, Mass., 1995.

Moore, T. E., et al., The thermal ion dynamics experiment and plasma source instrument, in The Global Geospace Mission, edited by C. Russell, pp. 429-439, Kluwer Acad., Norwell, Mass., 1995.

Paterson, W. R., L. A. Frank, S. Kokubun, and T. Yamamoto, Geotail observations of current systems in the plasma sheet, in Geospace Mass and Energy Flow: Results From the International SolarTerrestrial Physics Program, Geophys. Monogr. Ser., vol. 104, edited by J. L. Horwitz, D. L. Gallagher, and W. K. Peterson, 201-211, AGU, Washington, D. C., 1998.

Pedersen, A., Solar wind and magnetosphere plasma diagnostics by spacecraft electrostatic potential measurements, Ann. Geophys., 13, 118-129, 1995.

Press, W. H., S. Teukolsky, W. T. Vettering, and B. Flannery, Numerical Recipes, 2nd ed., Cambridge Univ. Press, New York, 1992.

Rosenbauer, H., H. Miggenreider, M. D. Montgomery, and R. Schwenn, Preliminary results of the helios plasma measurements, in Physics of Solar Planetary Environments, edited by D. J. Williams, pp. 319-331, AGU, Washington, D. C., 1976.

Scudder, J. D., E. S. Sittler Jr., and H. S. Bridge, A survey of the plasma electron environment of Jupiter: Voyager 1, J. Geophys. Res., 86, 8157-8179, 1981.

Scudder, J. D., et al., Hydra-A 3-dimensional electron and ion hot plasma instrument for the Polar spacecraft of the GGS mission, in The Global Geospace Mission, edited by C. Russell, pp. 459-495, Kluwer Acad., Norwell, Mass., 1995.

Whipple, E., J. Warnock, and R. H. Winkler, Effect of satellite potential on direct ion density measurements through the plasmapause, $J$. Geophys. Res., 79, 179-186, 1974.

Whipple, E. C., Potentials of surfaces in space, Rep. Prog. Phys., 44, 1197-1250, 1981.

J. D. Scudder and X. Cao, University of Iowa, Department of Physics and Astronomy, Iowa City, IA 52242. (jds@space-theory.physics. uiowa.edu)

F. S. Mozer, Space Sciences Laboratory, University of California, Berkeley, CA 94720.

(Received February 25, 1999; revised September 13, 1999; accepted September 13, 1999.) 